\title{
Philosophiques
}

\section{L’amitié, la société et la femme}

\section{John R. Gallup}

Volume 21, numéro 2, automne 1994

Les femmes et la société nouvelle

URI : https://id.erudit.org/iderudit/027283ar

DOI : https://doi.org/10.7202/027283ar

Aller au sommaire du numéro

Éditeur(s)

Société de philosophie du Québec

ISSN

0316-2923 (imprimé)

1492-1391 (numérique)

Découvrir la revue

Citer ce document

Gallup, J. R. (1994). L’amitié, la société et la femme. Philosophiques, 21(2), 417-431. https://doi.org/10.7202/027283ar
Résumé de l'article

Notre objectif est de montrer comment les principes mis de l'avant par Aristote, dans sa théorie de l'amitié, pourraient contribuer à l'avènement d'une société meilleure autant pour les femmes que pour les hommes. d'utilisation que vous pouvez consulter en ligne.

https://apropos.erudit.org/fr/usagers/politique-dutilisation/ 


\title{
L'amitié, la société et la femme
}

\author{
par \\ John R. Gallup
}

\begin{abstract}
RESUME : Notre objectif est de montrer comment les principes mis de l'avant par Aristote, dans sa théorie de l'amitié, pourraient contribuer à l'avenement d'une société meilleure autant pour les fermmes que pour les hommes.
\end{abstract}

ABSTRACT : Our aim is to show how Aristotle's theory of friendship is a contribution to the coming of the good society for women as well as for men.

\section{Introduction : Le rôle de l'amitié dans la société 1.I la vie humaine et le besoin de l'amitié}

Puisque ce colloque propose une discussion sur les femmes et la société nouvelle - et quej'ai la conviction que l'amitié humaine a un rôle important à jouer dans l'amélioration de la société actuelle - je présenterai un survol rapide de la nature de l'amitié telle que dégagée par les techniques d'observation appliquées à un monde existentiel et réel. Ces techniques, découvertes par Socrate, furent continuées par Platon et aussi par Aristote, qui fut pendant sept ans l'élève, et pendant dix ans, le collègue de Platon.

J'utiliserai les techniques de définition qu'emploie finement Aristote dans les livres VIII et IX de son Ethique à Nicomaque, où l'égalité et les caractères distincts des êtres humains mâle et femelle sont rediscutés à travers les analyses de la définition de l'amitié et de son rôle dans la vie humaine. Ces textes aident à mieux comprendre maintes observations fines sur l'amitié, que l'on trouve éparpillées dans divers textes littéraires d'autres cultures mais, de manière spéciale, dans les écrits du célèbre écrivain français Antoine de SaintExupéry. Cette tradition met en valeur l'égalité des individus en tant qu'êtres humains adultes, conscients et de bonne volonté.

Saint-Exupéry ne peut concevoir la société de demain sans deux ingrédients : l'amitié et la connaissance. En effet, comme l'a dit le Petit Prince : « Je cherche des amis. [...] J'ai des amis à découvrir et beaucoup de choses à connaître. » Apparemment, connaître est au-dessus de savoir. J'ai bon espoir 
que nous ne sommes toujours pas insensibles aux reproches que le renard fait à certains êtres humains :

On ne connait que les choses que l'on apprivoise, dit le renard. Les hommes let les femmes] n'ont plus le temps de rien connaître. Ils achètent des choses toutes faites chez les marchands. Mais comme il n'existe point de marchands d'amis, les hommes n'ont plus d'amis. Si tu veux un ami, apprivoise-moi ${ }^{\mathrm{I}}$ !

Même aujourd'hui dans notre société de consommation, ni l'amitié, ni la justice légale ne sont des produits achetables. Et il semble qu'il n'y aura pas de société nouvelle merureure s'il n'y a pas un effort plus grand d'apprivoisement de l'autre pour se faire des ami(e)s et bâtir la justice sociale. Les êtres humains doivent s'engager à s'apprivoiser. Atteindre la fin qu'est l'amitié et atteindre les fins de la justice sociale sont deux activités différentes qui doivent se compléter pour exister.

\section{2 la vie humaine et la justice légale}

Comme il est facile de l'observer, une société moderne et complexe exige une grande diversité non hétéroclite mais hétérogène dans les fonctions à remplir. Ces rôles sont fixés, en partie, par des lois positives provenant d'un système légal temporairement en vigueur. Cependant, avant que l'État ait commencé à faire ses lois, les communautés moins complexes se formaient suivant les besoins de chaque groupe et suivant les décisions des individus du groupe dans le but d'atteindre la survie commune. Certaines de ces décisions ont été transformées en habitudes et mœurs acquises avant de devenir formellement des lois. Comme conséquence, une partie de la vie du citoyen moderne est dirigée et systématisée par les lois positives héritées du passé.

\subsection{Une partie de la vie est dirigée par la loi positive}

Entre la vie civile des citoyens et des citoyennes et leur vie privée, il y a un écart. Le succès de la vie individuelle dépend de la manière de vivre sur les deux côtés de cette limite. Toute la vie de l'individu ne doit pas être dominée ou circonscrite au-dedans des restrictions de la loi positive d'un État particulier. La vie privée se trouve à un autre niveau; elle est nécessairement prélégale.

Ceci est vrai pour deux raisons. Tout d'abord, la raison d'être de la loi positive est d'aider les gens à augmenter la justice entre les citoyens selon ce qui est leur dû en tant que citoyens; mais la loi positive les attend souvent avec un juge mandaté pour trancher le point en litige. Chaque cas d'injustice est apte à être jugé selon une loi qui, étant faite pour la majorité des cas, est générale, même si dans une situation donnée l'application littérale causerait une plus grande injustice. Alors, le juge devrait appliquer le principe d'équité, en interprétant l'intention du législateur. Voilà deux aspects différents qui

I. Antoine de Saint-Exupéry, Le Petit Prince, édité dans CEuvres, Bibliothèque de la Pléiade, Gallimard, Paris, 1957, p. 470-471. L'ouvrage fut publié pour la première fois à New York, en février 1943, pendant la guerre. 
rendent la vie concrète très compliquée : I) La loi positive ne peut jamais garantir la justice pour tous les citoyens; la loi positive ne peut que viser l'atteinte d'un bien commun partagé par la plupart des citoyens. En conséquence, l'État devrait mieux prévoir le mécanisme secondaire d'équité afin de compenser les éléments d'injustice imbriqués dans la loi positive. iI) Cependant, on ne peut remédier à toutes les situations injustes même par la voie du principe de l'équité. La réalité est trop complexe puisque les lois positives sont toujours faites par tel État particulier, qui existe de manière limitée dans l'espace et dans le temps, et puisque même si les lois sont faites pour atteindre le bien commun, elles ne peuvent viser qu'un bien commun particulier à tel Etat particulier. Le mouvement féministe est né de la nécessité que les lois positives soient assujetties à des révisions constantes. Mais puisque les forces gouvernementales sont intrinsèquement archaïsantes, elles restent attachées comme des grippe-sous au pouvoir acquis, parce que formalisées et concrétisées par la loi, et elles sont lentes à changer les lois, même quand les lois sont devenues désuètes, pour ne pas dire injustes, pour une nouvelle génération de citoyens.

La deuxième raison pour l'importance de cette vie prélégale repose sur le fait que dans leur vie les individus n'ont pas besoin tout simplement de la justice, mais aussi de l'amitié. Dans la vie, l'amitié est à la justice légale, ce que la qualité est à la quantité. Étant par sa nature fort différente de la justice, l'amitié est un de ces besoins non négligeables de la vie, qui est, malheureusement, trop facile à négliger. Pourquoi ? Parce qu'elle ne peut jamais être contrôlée ni dirigée par les facteurs de rationalisation et de quantification si essentiels à la fabrication des lois justes. Quand les Etats essayent par leurs lois positives de contrôler l'amitié, des effets désastreux s'en suivent ${ }^{2}$. C'est l'amitié qui délimite le pouvoir limité de l'État. Si l'amitié n'existait pas, le pouvoir de l'État serait un monstre déchaîné, nécessairement totalitaire, pour ne pas dire férocement tout-puissant. Dans cette veine de pensée, Aristote a observé :

2. Dans le roman 1984 de George Orwell, l'amour sexuel avec la liberté de tomber amoureux est présenté comme l'élément destructeur de l'Etat totalitaire. Voir Michel Lavoie, « Premier fondement du pouvoir totalitaire : l'opposition Femme/ Homme dans 1984 de George Orwell », dans Les Cahiers $d u$ GRAD, nं 3. Misogynie, sexisme, féminisme : Images Ambiguës, Faculté de philosophie, Université Laval, ig8g. p. 105-I26. En effet, c'est la liberté de l'être humain individuel manifestée à travers l'amour, qui est l'ennemie perfide pour l'Etat totalitaire. La liberté personnelle manifestée à travers l'amitié est encore plus perfide, car la liberté de l'amitié est liée à la recherche de la vérité. En effet, l'État ne peut jamais posséder la vérité. Seuls les individus peuvent la posséder ! Dans sa nature profonde, l'État est là seulement pour pourvoir les individus des moyens qui permettent de continuer dans la prise de possession de la vérité. La temporalité de l'État, liée à son incapacité à posséder la vérité, fait que, quand il est dirigé par les hommes imprudents, il devient une bête, un animal sans raison. 
On pourrait même aller jusqu'à dire que c'est l'amitié qui est le lien des Etats, et que les législateurs s'en occupent ${ }^{3}$ avec plus de sollicitude encore que de la justice. La concorde des citoyens n'est pas sans ressemblance avec l'amitié; et c'est la concorde avant tout que les lois veulent établir, comme elles veulent avant tout bannir la discorde, qui est la plus fatale ennemie de la cité. Quand les hommes lles êtres humainsl s'aiment entre eux ${ }^{4}$, il n'est plus besoin de justice. Mais ils ont beau être justes, ils ont encore besoin de l'amitié5.

\subsection{Incadrée par l'amitié, la vie trouve sa juste valeur}

Pour rester fidèle à sa méthode de comparaison qui vise une compréhension complète du comportement humain, Aristote introduit un rapport mesure/mesuré entre l'amitié et la justice. Cette comparaison, qui semble étrange, m’a stimulé à présenter cet essai : « L'amitié, dans chacun de ces États ou gouvernements, règne dans la même mesure que la justice $»^{6}$. Cette comparaison qualitative mais scientifique présuppose une analyse de la justice déjà présentée au livre 5 ; et elle permet de faire ressortir les ressemblances et les différences entre la nature et l'importance relative des deux. Certes, Aristote est à la recherche de la preuve de la vérité que nous « avons beau être justes, nous avons quand même besoin de l'amitié ». Pour le présent essai, cette analyse est présumée déjà faite.

\section{La découverte de la définition de l'amitié}

\subsection{1'amitié est composée de trois âléments d'égalité}

La recherche d'Aristote a révélé que l'amitié est composée de trois éléments qui se situent respectivement au niveau i) de la nature commune des individus; 2) de la connaissance; et 3) de la bonne volonté.

\section{2l'égalité au niveau de la nature humaine}

L'amitié implique l'existence actuelle de deux êtres amicaux : la coexistence réelle d'au moins deux êtres naturels physiquement distincts ${ }^{7}$. Évidemment, il n'est pas question d'amitié, par exemple, entre moi et mon ombre, ni entre moi

3. Aujourd'hui, il écrirait peut-être : devraient s'en occuper !

4. Dans sa note 4, p. 312, J. Barthélemy Saint-Hilaire commente : « Admirables doctrines, qui semblent devancer déjà le christianisme, et qu'Aristote emprunte aux enseignements de son maître », Aristote, Morale d'Aristote, Livre VIII, chap. I, trad. par J. Barthélemy Saint-Hilaire, Paris, Durand, 1856 . J'utilise cette traduction de préférence à celle de Tricot, car elle respecte mieux en plusieurs passages l'esprit d'Aristote au sujet du rôle que joue la connaissance sensible dans l'amitié humaine.

5. Ibid., chap. I, p. 3II [Bekker, I155 a22-28], traduit par J. Barthélemy Saint-Hilaire, Paris, Durand, 1856 .

6. Ibid., chap XI, p. 350 [Bekker, chap. I3, II6I aı-IIl.

7. Cette section semblerait tricotée de banalités, mais ce qui est important est la coordination totale; une comparaison facile serait de regarder les ingrédients banals d'une recette, qui, une fois bien mélangés, donnent un gâteau extraordinaire. 
et une statue de moi ! L'ombre, c'est une absence de réalité; et la statue, un produit de l'art. Entre deux êtres matériels, il existe des rapports, des relations, d'inégalité ou d'égalité. On constate facilement l'inégalité entre moi et mon ombre, entre moi et ma statue, qui rend l'amitié impossible même si j'aime mon ombre ou la belle statue de moi-même.

Les êtres humains sont de par leur nature dotés de cinq caractéristiques réelles dont il faut tenir compte pour exposer la sorte d'égalité requise pour l'amitiè. En rabâchant les soi-disant banalités connues, à savoir, que nous, les êtres humains, sommes I) des substances matérielles, 2) des vivants, 3) aussi des animaux, qui sont dotés 4) de la capacité de parler et de raisonner, et 5) d'une sexualité féminine ou masculine, nous avons un cadre adéquat pour poser cinq questions dont les réponses fraient le chemin à la recherche. En bref, quelle sorte d'amitié réelle peut relier les êtres humains l) avec les autres êtres naturels non vivants, 2) avec les autres êtres vivants, 3) avec les autres animaux, 4) entre eux et 5 ) entre eux en tenant compte de la distinction sexuelle?

Pour la première des cinq questions, il faut répondre que nous aimons les êtres matériels, mais pas par un amour d'amitié. L'argument que les meilleurs amis des êtres humains sont les biens matériels, c'est seulement une métaphore poétique, n'est-ce pas ? En effet, nous sourions en écoutant les paroles d'une chanson américaine, chantée par Marilyn Monroe et d'autres, Diamonds are a girl's best friend : nous y reconnaissons une métaphore qui rend sensible au fait que les vrais amis doivent être fiables et durables. L'égalité seulement au niveau le plus général de notre nature matérielle n'est pas l'égalité sur laquelle se fonde l'amour par amitié. Nous aimons les choses matérielles inanimées surtout comme moyens utilitaires ordonnés à une fin du sujet qui est soimême. On vendra ses amis les diamants, quand on aura besoin d'argent pour acheter de quoi manger.

Le royaume des plantes est composé des substances matérielles vivantes fonctionnant selon les trois capacités : I) de se nourrir, 2) de croître pour devenir adulte, et 3) de reproduire d'autres individus de la même espèce. Par le biais de ces trois fonctions qu'ils possèdent aussi, les êtres humains jouissent d'une plus grande égalité avec les plantes qu'avec le monde minéral. Les plantes occupent une grande place dans notre vie. Ainsi la culture la plus nécessaire aux hommes, c'est l'agri-culture. Aucune société ne peut survivre sans la culture de sa nourriture. Nous nous occupons des plantes surtout comme de grands moyens de nous nourrir. Nous les aimons, parce qu'elles sont bonnes à manger. Nous les aimons, mais quand nous les mangeons pour survivre, nous les détruisons. Ça, ce n'est pas l'amour par amitié. L'amitié n'est pas destructrice de l'ami. Par ailleurs, même quand nous cultivons souvent des plantes avec amour, attention et affection, il n'y a pas de réciprocité de connaissance et d'affection.

Quand, à ce moment, nous parlons des animaux, nous respectons le sens scientifique et traditionnel de ce mot tel qu'utilisé en zoologie depuis les Grecs : à savoir qu'un animal est une substance matérielle vivante ayant 
comme caractéristique déterminante au moins un organe requis pour faire des actes de connaissance sensible selon le toucher. Par cette connaissance sensible, les animaux, les hommes inclus, peuvent d'abord mieux chercher leur nourriture tandis qu'au contraire les plantes entrent en contact et assimilent leur nourriture sans en prendre connaissance. Donc, pour tous les animaux, la connaissance sensible est d'abord et restera nécessairement une activité utile pour protéger la survie de l'individu et, à long terme, l'espèce. Selon l'ordre d'une progression vers le mieux, la connaissance comme activité propre à certains êtres vivants a réussi à se faire intérioriser ou intégrer comme découlant d'une capacité inhérente à leur nature.

L'homme est un animal précisément parce qu'il possède la condition minimale de la connaissance sensible par le toucher, mais il possède beaucoup plus, à savoir toute la gamme des cinq sens externes et des quatre sens internes, qui s'achève avec le pouvoir estimatif animal et son trésorier, la mémoire du passé. Ces deux dernières puissances apportent la connaissance de ce qui est bon ou mauvais pour le sujet vivant qui est appelé à protéger sa vie et sa santé ${ }^{-8}$. Par ailleurs, c'est l'importance primordiale du sens du toucher qui fonde le besoin de donner et de recevoir, par le contact physique, de l'affection. Chez les êtres humains, l'amitié inclut toujours la connaissance et l'affection sensibles.

Quand selon le proverbe on dit que le chien est le meilleur ami de l'homme, on reconnaît le fait que les deux êtres se connaissent, se reconnaissent, s'aiment, et partagent de manière visible de l'affection. Nous constatons de manière spéciale que le degré d'égalité des hommes avec les autres animaux est plus intense qu'avec les plantes, ou avec les substances matérielles non vivantes. Leur nature essentielle est plus identique, et cette identité réside dans la connaissance sensible et le besoin de donner et recevoir l'affection. Néanmoins, puisque la reproduction sexuelle apparaît déjà chez certaines plantes et se retrouve dans la plupart des espèces animales, il est clair que le rapport entre la sexualité et la connaissance est un rapport accidentel ${ }^{9}$, pour ne pas dire, étrange. Cependant, le problème sexuel chez les êtres humains se noue autour du lien entre la connaissance sensible tactile et le plaisir du

8. C'est par cette phrase que je rattache tout ce qui est important dans les découvertes de Freud à la tradition aristotélicienne. Freud a redécouvert ce qui, au moyen âge, s'appelait la vis astimativa ou cogitativa, le pouvoir estimatif du bien et du mal biologique. Il est connu que Freud avait suivi un cours sur la logique d'Aristote.

9. C'est ici que nous retrouvons la grande difficulté pour celui qui fait du besoin sexuel le besoin le plus fondamental chez les êtres humains. Il n'est le besoin fondamental d'aucun autre être vivant. Toutes les théories de l'amitié tournent nécessairement autour de ce problème. Et il faut une méthodologie de travail sans failles pour trouver la bonne réponse. 
contact sexuel. Le lien, accidentel et réel, ouvre la porte à des problèmes réellement épineux.

Finalement, parmi les êtres vivants qui sont des animaux, il y a les humains, ceux qui sont dotés de poumons, d'un larynx, mais surtout d'une langue assez déliée pour être capable de prononcer une série indéfinie de consonnes et de voyelles. En plus d'un besoin d'utiliser des sons vocaux comme signes naturels, ils doivent organiser et inventer divers signes conventionnels. Parmi ces symboles, les plus remarquables sont les mots par lesquels nous manifestons non seulement notre connaissance et nos émotions sensibles, mais aussi notre connaissance intellectuelle et notre capacité de raisonner $^{\text {Io }}$. L'homme est la seule espèce animale capable d'être bilingue, transcendant partiellement l'esclavage des signes naturels qui domine et retient les autres animaux!

Tous les êtres humains sont dotés essentiellement des capacités intellectuelles invisibles. Et c'est dans la différence spécifique qui sépare l'homme des autres espèces d'animaux que nous retrouvons le fondement de l'égalité qui rend l'amitié une chose possible. La vraie amitié ne peut exister qu'entre les êtres qui jouissent de la connaissance intellectuelle et de sa suite, la volonté libre ${ }^{\mathrm{II}}$.

\subsection{La connaissance mutuelle et l'égalité au niveau de la connaissance}

L'amitié n'est pas simplement basée sur une nature; elle est liée au comportement humain, à des actes de connaissance. Les Grecs avançaient dans l'analyse des égalités requises, en constatant que les êtres humains doivent se connaitre mutuellement. Chaque ami doit sentir et savoir que l'autre est son ami. L'ignorance n'est pas admise. L'amitié, comme activité de connaissance, embrasse des connaissances sensibles avec certaines affections qui en découlent aussi bien que la connaissance intellectuelle. Tandis que l'amour

10. À cause de la structure grammaticale du verbe français, le mot raisonner signifie de manière systématique soit être momentanément en train de raisonner soit avoir la capacité de raisonner même si on n'est pas en train de raisonner. Raisonner implique, d'abord, l'activité de compter et aussi de s'en rendre compte. L'être humain est le seul animal comptable, capable de compter et de s'en rendre compte. En utilisant le mot comptable, je voudrais rappeler le fait que le mot latin ratio (nom d'action, dérivé du verbe reor, reri, ratus sum) d'où dérivent les mots raison et raisonnable, signifie l'activité ou la capacité de compter. Bien raisonner, bien compter, se rendre compte correctement, ce sont des activités des êtres humains qui peuvent mettre les parties ensemble, réussir la formation de touts cohérents et finalement évaluer cette cohérence.

II. Comme il est facile de l'observer, la contrarièté mâle-femelle ne constitue jamais des espèces biologiques; en effet, c'est une contrariété qui arrive en dehors de et à côté de - la classification selon les différences essentielles constituant l'une ou l'autre espèce. Il arrive que certains êtres sexués sont des plantes, et d'autres. 
sexuel ou passionnel peut être unilatéral (en effet, prise d'un tel amour, une personne peut même vouloir forcer l'autre à l'aimer, contre sa volonté), l'amitié n'est pas comme ça.

Chaque être humain a son monde intérieur et vit la vie voilée de ses développements émotionnel et cognitif intérieurs. Nous avons chacun nos rêves et désirs secrets. Dans l'épanouissement de l'amitié, les amis sont attirés tranquillement à faire la révélation de leurs secrets intérieurs. Sans cela, ils ne se connaissent pas suffisamment bien. La vraie amitié veut atteindre une égalité de connaissance, pas nécessairement une égalité entre les savoirs.

Comme l'a remarqué Alexandre Manzoni ${ }^{12}$, un des plus grands bonheurs de cette vie, c'est l'amitié; l'un des bonheurs de l'amitié, c'est d'avoir à qui confier un secret. Révéler aux autres ses secrets, c'est dire ses vérités aux autres. Ce niveau d'analyse fait comprendre que l'amitié est une propriété qui se loge chez les animaux qui sont capables de chercher, de posséder et de dire la vérité. De manière spontanée, on n'accepte pas comme amis ceux qui nous mentent ni ceux qui ne gardent pas nos confidences. Il ne faut pas dire nos secrets à n'importe qui, mais seulement à ceux à qui on fait confiance. La confiance, présupposant l'existence de la vérité, voilà une autre propriété imbriquée dans l'égalité amicale.

\section{4 la bonne volonté envers l'autre comme à un autre soi : l'égalité au niveaul de la bonne volonté}

Chez tous les animaux, la connaissance est suivie de l'une ou l'autre des deux réactions émotionnelles possibles. Si la chose connue est estimée bonne par tel animal, la suite émotive est celle d'un amour, d'une attirance vers l'objet, d'un désir de le posséder et d'une jouissance spéciale dans sa présence; par contre, si la chose connue est estimée mauvaise pour et par lui, la suite émotive est celle de la haine et de la fuite de sa présence. La chose évaluée comme bonne l'attire. De plus, chez les êtres humains, la volonté est attirée vers les choses évaluées comme bonnes. Et dans l'amitié, le partage de la vérité est vu comme bon. Cependant, les choses qui nous attirent comme un moyen utile pour une

des animaux. Un être mâle peut être seulement une plante, ou un animal, et si c'est un animal, un être doté seulement de la connaissance sensible ou, en plus, de la capacité d'une connaissance universelle. Qu'un être humain soit un mâle est. selon un sens réel du mot, accidentel. Cependant, cet accident appartient à la catégorie de la substance, car les organes sexuels sont les parties intégrales substantielles du corps vivant. Ce n'est pas un accident tel que la quantité ou la qualité. C'est pourquoi le mot femme, en contraste avec le mot femelle, signifie un être qui est essentiellement un être humain comportant des spécifications (accidentelles à la nature spécifique, mais requises pour la génération) quant à la nature des parties intégrales corporelles. La recherche moderne ne peut rien ajouter à l'analyse fine apportée sur ce problème par Aristote, Métaphysique, livie ro (ou I), chap. 9, [Bekker 1058a29-b25] sauf le fait que la distinction sexuelle se trouve aussi chez certaines plantes supérieures.

12. Dans son roman Les fiancés (chap XI). 
fin, forment le domaine de la plupart de nos rapports sociaux. Le maître de maison cherche, comme un moyen, un homme-à-tout-faire. Le directeur d'une compagnie cherche les êtres humains compétents pour atteindre ses propres fins. De manière tout à fait morale, la volonté d'un être humain est attirée vers d'autres personnes comme vers des moyens utiles en vue d'une fin. Mais le fondement de l'amitié ne se trouve pas là, car dans ces cas-là, l'autre est subordonné et est traité comme un inférieur par le premier. En effet, dans une société complexe, la diversité des tâches à accomplir entraîne des inégalités dans les rangs sociaux. La seule manière par laquelle un être humain peut être attiré par un autre de manière égale, c'est d'aller vers lui comme vers un autre soi, un égal. Mais puisque je ne suis pas un moyen par rapport à moi-même, mon amour de moi-même, est un amour vers un être comme vers une fin. Donc, aimer un autre comme un autre soi, c'est l'aimer comme une fin, avec toute la dignité que cela comporte. La bonne volonté requise pour l'amitié, c'est l'amour envers un autre être humain, l'amour envers un être attirant, comme seulement une fin peut attirer. Par contraste, une cause efficiente tire, elle n'attire pas; et elle peut même tirer de manière violente tandis que la fin ne peut que nous attirer. L'attraction de la cause finale n'est jamais violente. Et dans l'amitié, deux êtres s'attirent comme des biens qui sont des fins. L'amour de l'amitié se réalise sous la condition que chaque ami qui aime est aussi aimé comme une fin. Dans cet amour, l'ami aime l'aimé sans se subordonner à lui comme s'il était un moyen à une fin. Cet amour découle d'actes libres des deux côtés. C'est pourquoi il est quelque chose hors du pouvoir de l'État, voire destructeur de l'État tyrannique. La vraie amitié prélégale sera constructrice et en quête de justice sociale.

L'amour passionné ou sexuel ne trouve pas là sa juste place; il se trouve à côté de ça. Un tel amour traite l'autre comme un moyen en vue d'une fin. Il trouve son complément dans la connaissance selon le sens du toucher et le plaisir que cela procure. Le regard sexuel est aussi subordonné au sens du toucher, comme un moyen à une fin. Pour mieux comprendre que l'amitié est différente, on peut adapter en les paraphrasant les paroles célèbres de SaintExupéry : « Les amis ne sont pas ceux qui se regardent les uns les autres, mais ceux qui regardent dans la même direction $\gg^{{ }^{3}}$. Regarder dans la même direction, c'est la métaphore poétique par laquelle Saint-Exupéry indique l'égalité entre les amis qui s'aiment. L'amour par amitié ne s'identifie pas avec l'amour sexuel, il est antérieur au cadre contraignant de la contrarièté sexuelle. Aimer

13. « Liés à nos frères par un but commun et qui se situe en dehors de nous, alors seulement nous respirons et l'expérience nous montre qu'aimer ce n'est point nous regarder l'un l'autre, mais regarder ensemble dans la même direction. Il n'est de camarades que s'ils s'unissent dans la même cordée, vers le même sommet en quoi ils se retrouvent. » Antoine de Saint-Exupéry. Terre des Hommes, dans CEuvres, op.cit., p. 252. 
par amitié peut coexister avec, ou exister indépendamment, de l'amour sexuel $^{14}$.

\section{5 les trois eléments de la définition réunis}

L'amitié, une qualité que les êtres humains peuvent acquérir et développer sans cesse ${ }^{\mathrm{I5}}$, implique l'intégration de trois égalités, dont l'une est au niveau de la nature humaine présupposée dans les sujets ${ }^{16}$ aptes à posséder la qualité amicale, et les deux autres sont au niveau des activités de l'intelligence et de la volonté qui entrent en activité réciproque. Du point de vue de l'art de définir, le genre est: « qualité acquise par un être humain en rapport avec un autre être humain »; la première différence : « ayant une connaissance réciproque (égale) »; la dernière différence : « ayant une bonne volonté réciproque (égale) $\gg^{17}$.

Aimer par amitié, ce n'est pas aimer d'un amour situé essentiellement dans la matérialité de l'être et de la survie biologique. Cette activité présuppose plus que l'élément fondamental de la possession de la même nature vivante. Dotée évidemment de la connaissance sensible et des émotions, l'amitié humaine doit être chaleureuse. Elle exige une curiosité envers d'autres humains et cherche une connaissance intellectuelle en toute bonne volonté. Elle fait développer une connaissance mutuelle, de plus en plus égale; et elle pousse la volonté des amis à se laisser attirer l'un par l'autre comme par une fin. Tandis que la science cherche le savoir universel, l'amitié vise à connaître l'individu qui peut connaître la vérité sans nécessairement être un homme de savoir.

Dans un sens, on ne peut que s'aimer soi-même. Ça ne veut pas dire que l'on aime seulement soi-même. Mais l'amour que l'on a pour soi-même est la mesure de l'amour que nous avons envers les autres. Si donc une personne s'aime, il arrive qu'elle peut s'aimer bien ou mal. La qualité, la manière de s'aimer soi-même est la mesure de la disponibilité à se faire des amis. Car si l'on s'aime mal, on s'empêche de traiter l'autre comme un égal.

14. Evidemment, une théorie de l'amitié basée sur les principes de Freud, principes qui sont de plus en plus mis en doute par la recherche scientifique, ne pourrait accepter une telle thèse.

15. Elle est même nécessaire pour le plein épanouissement de la personnalité de l'individu. Cet aspect n'est pas étudié dans cet essai.

16. Selon l'art de la définition, le genre provient de la nature humaine, donné par les lois de l'hérédité aux individus qui agissent. La nature humaine est donc une condition nécessaire préalable, un catalyseur qui permet que les actes de connaissance et de volonté fonctionnent en respectant la liberté de l'autre.

17. Dans une autre discussion étrangère à notre but précis, il faudrait en plus observer les différentes espèces d'amitié. 
Dans un livre exceptionnel sur l'amitié, Les chemins de l'amitié ${ }^{18}$, le psychologue et auteur français Ignace Lepp commence son étude du besoin humain de l'amitié en s'opposant à la description de la nature humaine proposée par Jean-Paul Sartre, que l'homme serait essentiellement un être esseulé ${ }^{19}$. D'après lui, l'esseulement consiste dans le fait qu'un être humain n'est capable ni d'aimer ni d'être aimé. Il est incapable d'aimer un autre ou lui-même tout seul; incapable d'être aimé par un autre ou par lui-même. Cet être qu'est l'être humain n'est ni sujet ni objet d'amour. La nature de l'homme, ainsi stérilisé, ou châtré, par la double absence de la puissance active et passive, est incapable d'aimer et incapable d'être aimé. Selon ce point de vue, chaque être humain est ainsi, (pour ceux qui veulent voir clair), son propre enfer avant d'être un enfer pour l'autre. Voilà ce que serait la nouvelle société des hommes et des femmes sans l'amitié. Selon une autre image, quand deux êtres humains sont face à face, ils sont comme deux miroirs cassés s'opposant dans la noirceur. Le néant de l'intériorité, en effet, c'est quelque chose d'esseulant et de désespérant!

\section{L'activité principale de l'amitié : parler ensemble}

Il apparaît étrange de le dire, mais l'activité principale de l'amitié semble être celle de se parler l'un à l'autre. Se parler exige à la fois une distance et un respect, car il faut aussi écouter. L'analyse de cette activité exigera beaucoup de temps. Qu'il suffise de dire que parler implique dans ce contexte la tâche délicate de ne pas mentir, de ne pas briser la confiance et de connaître profondément l'autre soi qui n'est pas encore suffisamment connu. Peut-être que notre colloque est un exemple d'amitié: nous sommes tous venus partager des idées comme pour poser des gestes amicaux, comme des philosophes ${ }^{20}$ qui

I8. Le titre du premier chapitre suggère le problème: Solitude et déracinement de l'homme modeme. On y lit : « C'est probablement parce que certains philosophes et leurs disciples ne connaissent que ce genre d'interminables discussions, dans une salle de réunion ou autour d'une table de café, sur la politique, la littérature et l'art, qu'ils croient devoir affirmer l'impossibilité de l'homme de sortir de son esseulement. C'est parce que l'être humain est capable d'aimer et d'être aimé que l'esseulement ne constitue point la fatalité de notre condition dans le monde ». Ignace Lepp, Les chemins de l'amitié, Paris, Grasset, 1964, p. 27.

19. EsSEurÉ, p.p. et adj. Qui est seul, abandonné. V. DÉlaissÉ, ISOlÉ, SEul, SOLITAIrE. Jeune fille esseulée dans un coin du bal. « Mais comme elle [Madeleinel était la seule, dans son endroit, qui n'eût pas du tout souci d'elle-même, elle se trouvait bien esseulée et s'ennuyait beaucoup, sans trop connaître la cause de son ennui. » (G. Sand, François le Champi, II, p. 37). dans Le Robert, Tome 2, Paris, I966, p. 645.

20. Basé sur le grec, ce mot pourrait être élégamment remplacé par un mot construit sur la manière française de former les mots : aime-sagesse. Comme le grec, le français forme souvent les mots à partir d'un verbe suivi par son complément, par exemple, casse-cou, coupe-papier. L'activité première de l'aime-sagesse est d'aimer la sagesse et par la suite de s'employer à la chercher et à la posséder selon ses possibilités. 
aiment parler et partager leurs secrets avec d'autres êtres humains. Je crois que les êtres humains sont capables d'aimer et d'être amis, même si la réalisation n'est pas toujours facile. Je tiens à respecter l'égalité que nous avons en tant qu'êtres humains, qui sont accidentellement et existentiellement séparés, ou distanciés les uns des autres, en tant que mâles et femelles. Nous avons tous des vérités à rechercher, à découvrir ensemble, et à partager. L'une d'elles, c'est la priorité de notre nature humaine commune d'où découle la dignité de notre sexualité individuelle. Un ami doit parler avec son ami comme avec un égal, car si la finalité principale du langage humain est de permettre à chaque être humain, à chaque personne, de se parler d'abord à lui-même, il faut a fortiori aussi être capable de parler à l'autre comme à soi-même. La carapace de l'égoïsme malsain est de parler à un autre comme à un inégal, un inférieur.

Cependant, parler est toujours une activité entourée par le silence. Il faut assez de silence pour écouter la parole et la méditer; aussi est-il évident que chaque phrase est précédée et suivie d'un silence. Comme l'essentiel invisible, l'amitié dure, et peut même se manifester à travers les périodes de silence aussi bien que de conversation. Les amis reprennent sans gêne la conversation rapidement, même après un long intervalle de silence ou d'absence. Le silence est amical ou froid selon la chaleur de l'amitié, et l'ami prend plaisir à la présence même de l'ami quand il ne parle que métaphoriquement par sa présence physique.

\section{Son plaisir principal : prendre plaisir à la présence de l'autre}

Selon les analyses d'Aristote, posséder les grands moyens pour atteindre activement la fin de l'homme, c'est posséder les vertus. La fin de l'homme, le bonheur, comporte comme propriété le plaisir. Le plaisir de l'amitié est intense; donc pour trouver la place juste de l'amitié dans le schème moral, il faut avoir un critère pour juger si le plaisir inhérent dans l'amitié est celui propre aux moyens ou à la fin. Se faire des amis exige des efforts parfois pénibles; se faire vraiment des amis nous oblige à devenir de plus en plus justes, courageux, tempérants et prudents. Voilà pourquoi l'amour nous force à agir de manière juste, ce qui implique des dépenses d'énergie parfois très grandes. Le curieux de la position d'Aristote, c'est qu'un être humain ne peut rester ni juste, ni prudent, ni courageux, ni tempérant pendant longtemps sans être entouré par les amis. On doit pratiquer les vertus pour se faire des amis; on doit aimer les amis pour s'encourager dans la pratique des vertus. Mais l'amour de la fin est la source qui inspire l'amour des moyens. C'est le plaisir de la vraie amitié qui nous assure le système de protection pour agir comme un bon citoyen ${ }^{2 \mathrm{I}}$. Une société stable

21. Il faut pointer un doigt réprobateur vers ces aristotéliciens qui exposent la théorie morale sans une longue discussion sur la place de l'amitié comme le seul milieu où les êtres humains peuvent se développer moralement et correctement. Pas d'amitié, pas de société stable! 
exige la confection de lois de plus en plus justes, que les citoyens sont légalement obligés de suivre, mais par sa liberté interne l'amitié devance, dépasse et entoure les restrictions légales imposées aux citoyens pour atteindre la fin immédiate de l'État.

Pour mettre à sa juste place le plaisir que l'amitié nous procure, il faudrait le comparer au plaisir auquel l'homme a accès à cause de l'existence des biens matériels non vivants, des êtres vivants végétaux, des autres animaux, et finalement par l'utilisation des autres êtres humains. Mais selon le rapport de moyens-fin, nous avons vu que les êtres humains doivent respecter certains droits d'infériorité et de supériorité envers d'autres humains. Au-dedans et audehors de ce rapport, il y a nécessairement place pour que les humains se voient comme des égaux.

Il y a donc lieu de contraster deux égalités qu' implique la liberté humaine. Il y a, d'un côté, une égalité légale toujours à protéger par les lois. Il me semble que c'est cette égalité légalisante que Saint-Exupéry a raillée dans Citadelle:

Dans ta liberté tu heurtes le voisin et il te heurte. Et l'état de repos que tu trouves c'est l'état de billes mêlées quand elles ont cessé de se mouvoir. La liberté ainsi amène à l'égalité, et l'égalité amène à l'équilibre, qui est la mort $^{22}$.

[...] Je ne fonde point le respect de l'homme sur le partage de provisions dans une égalité haineuse l...] Tu trouveras l'égalité, une fois la marche inutile, à l'heure de la mort, quand Dieu engrange $e^{23}$.

Il y a, de l'autre côté, une égalité qualitativement différente, celle de l'amitié. Plus riche, plus fondamentale, elle est dans un sens plus facile à maintenir et dans un autre plus difficile. Cette égalité provient du fait que la nature a doté les individus d'une même nature spécifique. Donc elle découle évidemment de la loi naturelle, pas de la loi positive que les chartes de l'homme essaient d'exposer. Ce sont ces valeurs moralement plus fortes que les lois légales que les chartes essaient de défendre. En effet, il y a une liberté et une égalité naturelles plus réelles et durables que celles que l'État accorde, et dont les mauvais États peuvent empêcher la réalisation.

Néanmoins, même si son fondement est donné par la nature, la réalisation de l'amitié dans le vécu dépend des actes de connaissance et de bonne volonté que les individus posent.

Selon notre auteur grec, le plaisir propre à l'amitié se trouve devant l'existence de l'être aimé, mais surtout devant, et dans, la présence physique de l'ami. Aristote argumente à cet effet par une comparaison avec l'amour passionnel :

Et de même que les amants se plaisent passionnément à voir l'objet aimé, et qu'ils préfèrent cette sensation à toutes les autres, parce que c'est en elle surtout que consiste et se produit l'amour, de même aussi les amis ne

22. A. de Saint-Exupéry, op. cit., chap. XCII.

23. A. de Saint-Exupéry, op. cit., chap. CXCVIII. 
recherchent-ils pas par-dessus toutes choses à vivre ensemble ? L'amitié est une association; et ce qu'on est pour soi-même, on l'est pour son ami. Or, ce qu'on aime en soi personnellement, c'est de sentir qu'on est; et l'on se plaît à la MÊME sensation pour son ami. Mais ce sentiment n'agit et ne se réalise que dans la vie commune, et voilà comment les amis ont si fort raison de la désirer. L'occupation dont on fait sa propre vie, ou dans laquelle on trouve le plus de charme, est celle aussi que chacun veut faire partager à ses amis en vivant avec eux. Ainsi, les uns boivent et mangent ensemble; d'autres jouent ensemble; d'autres chassent ensemble; d'autres se livrent ensemble aux exercices du gymnase; d'autres s'appliquent ensemble aux études de la philosophie; tous en un mot passent leurs journées à faire ensemble ce qui les charme le plus dans la vie. Comme ils veulent vivre toujours avec des amis, ils recherchent et ils partagent toutes les occupations qui leur paraissent pouvoir augmenter cette intimité et cette vie commune. Tout instables qu'ils sont dans leurs affections, ils ne se communiquent que de mauvais sentiments; et ils se pervertissent d'autant plus qu'ils s'imitent mutuellement. Au contraire l'amitié des honnêtes gens, étant honnête comme elle l'est, ne fait que s'accroître par l'intimité. Ils semblent même s'améliorer encore en la continuant, et en se corrigeant.

\section{L'amitié et la société : seule une société encadrée par l'égalité propre de l'amitié peut protéger les droits de la femme}

Ma thèse est la suivante : La vie menée selon la loi positive trouvera sa juste valeur et son plein épanouissement seulement quand elle sera encadrée par un écosystème moral. Certes, la vie de citoyen doit être menée en respectant les droits et les obligations fixés par la loi positive, mais le citoyen suffisamment instruit comprendra que les lois sont toujours à renouveler et à améliorer. La loi positive n'existe jamais toute seule pour elle-même, étant seulement un moyen requis pour atteindre une certaine fin. Le prérequis pour vouloir obéir à la loi positive, c'est l'amour de la fin visée. Sans cet amour, la loi ne peut pas être librement acceptée par les citoyens; et s'ils la suivent sans amour, il la suivent par peur, donc comme esclaves accroupis devant un maître tyrannique. L'amour envers les moyens découle de l'amour préalable de la fin. Mais cet amour doit être fortifié par l'amitié.

Mais la dignité de la personne ne découle pas de la fin de l'État; elle la précède. Les citoyens individuels doivent aimer la fin de l'État comme un moyen d'atteindre, de respecter, d'aimer la dignité de la personne humaine. Voilà l'aspect moral que je voudrais appeler l'écosystème moral. La justice selon la loi positive ne peut être acceptée ni suivie sauf si elle est entourée, encadrée et subordonnée à la moralité où la volonté libre entre en jeu de manière responsable. Mais il y a plus : une distinction entre un système de moralité et un écosystème moral. Un système de moralité est incomplet, s'il ne prévoit pas la différence entre la fin d'un État particulier et l'amour de la personne individuelle, basé sur la dignité de la personne. L'écosystème moral commence avec l'amour qu'est l'amour de la dignité de la personne humaine, et ensuite il ouvre la porte à l'amitié. Quel rapport y a-t-il entre l'amour de la fin de l'État qui existe pour le bien commun des citoyens, et l'amitié qui peut exister entre les êtres humains? 
Cet écosystème moral est l'amitié entre les êtres humains. L'écosystème moral, dont l'existence est facile à observer, c'est celui où les êtres humains utilisent les moyens divers pour atteindre leurs fins diverses, avec la conscience de vouloir agir, et agir de manière juste. La justice, la soif de la justice, et la difficulté d'être juste surgissent chaque fois que les humains forment des sociétés pour atteindre une fin commune. C'est toujours l'amour de la fin des humains, lesquels nous attirent pour se servir des moyens humains pour l'atteindre. Où donc l'amitié se place-t-elle dans le rapport des moyens sociaux et de la fin sociale? Vraisemblablement, l'amitié fait partie intégrante de la fin de chaque être humain, et partie du bonheur de tous les êtres humains. Si la justice, entourée par la prudence, le courage, et la tempérance, est le moyen principal à employer pour atteindre le bonheur en société, l'amitié, comme situation où nous sommes de vrais amis, fait plutôt partie intégrante de la fin de l'homme. N'est-il pas vrai qu'il n'est jamais permis d'utiliser ni l'amitié ni l'ami comme un moyen en vue d'une fin ? Voilà, d'après moi, la position dégagée par les Grecs, et de manière éclatante par Aristote, que je propose comme considération toujours actuelle.

\section{Conclusion}

Sans faire d'effort pour encourager entre les êtres humains le développement d'amitiés qui devancent toutes les obligations en justice, il est difficile d'améliorer les démarches qui visent l'amélioration de nos systèmes de justice légale et la réduction des injustices entre les êtres humains, qui sont inévitablement des êtres sexués.

Si ces considérations sont valables, il sera nécessaire de laisser une place primordiale à l'amitié dans la vie saine et normale des hommes et des femmes dans le développement des stratégies que les mouvements féministes seront appelés à inventer dans le but d'améliorer les lois positives entachées par un excès d'injustice établie. En effet, chaque individu a des propriétés et des qualités qui sont antérieures aux droits et privilèges acquis par les lois positives. Autrement dit, si la transformation de certaines lois mal faites et source d'injustice est un médicament à prescrire, la contre-indication de cette médication, dont il faut être conscient, sera le danger de tomber dans un excès de légalisme. Un légalisme excessif brisera ou diminuera les possibilités du développement des vraies amitiés entre femme et femme, femme et homme, et homme et homme. En effet, un excès de lois positives deviendrait toujours un dragon déchaîné, une bête totalitaire destructrice de l'amitié. 